\title{
Development of a Cost-Efficient Computer Controlled Optical Surfacing Pro- cess for Correcting Aspheric Lenses using Tool Influence Function based Dwelltime Optimization
}

\author{
Pei Liang Low ${ }^{1, *}$, Wilhelmus A. C. M. Messelink ${ }^{1}$, and Rene Weber $^{1}$ \\ ${ }^{1}$ Edmund Optics Singapore Pte. Ltd., 18 Woodlands Loop \#04-00, Singapore, Singapore
}

\begin{abstract}
A Computer Controlled Optical Surfacing (CCOS) system has been developed for correcting form errors on aspheric surfaces. Experiments were carried out to find the correlation between different polishing parameters and polishing metrics such as removal rate, uniformity etc. Based on established polishing parameters, polishing process is developed to correct surface errors on planar, spherical and aspheric surfaces. A convolution model between TIF and dwell times was developed to simulate and solve for correction polishing. Surface accuracies of peak-to-valley (PV) $141 \mathrm{~nm}$ and root-mean-squared (RMS) $22 \mathrm{~nm}$ has been achieved for planar surface. For aspheric surface, current accuracy of $662 \mathrm{~nm}$ PV and of $115 \mathrm{~nm}$ RMS is achieved with further development ongoing.
\end{abstract}

\section{Introduction}

The global market is moving towards tighter and tighter specifications on aspheres, while at the same time pushing for a reduction in unit price. This trend has been ongoing for several years and Edmund Optics understands customers need for mid-range, price-sensitive aspheres, which requires an alternative corrective technology than the current, costly, state-of-the-art.

To improve the accuracy of a CNC polished asphere, it is typically 'finished' on a much more expensive, corrective polishing platform (such as Magneto-Rheological Finishing [1], Precessed Bonnet Polishing [2] or Ion-Beam Figuring [3]). These so-called Computer Controlled Optical Surfacing (CCOS) platforms are commercially available and in widespread use around the world, and are being installed more and more in low-cost countries. Using these platforms on the lower-end of their capability scale will therefore become a very competitive and uneconomical use of our machine park. This is summarized in the capability versus cost matrix in in Table 1 , where the red colour denotes an area of low economic value.

To fill the highlighted gap we have started the in-house development of a cost-efficient CCOS platform to serve the moderately precise asphere market. We achieve this by using proven, of-the-shelf components, flexible processes and existing industry standards.

\section{Approach}

In CCOS [4] the surface form error and the removal characteristics of the finishing process are measured and quantified, after which a computer algorithm calculates the process parameters to be used to correct the surface.
Table 1. Capability versus cost matrix

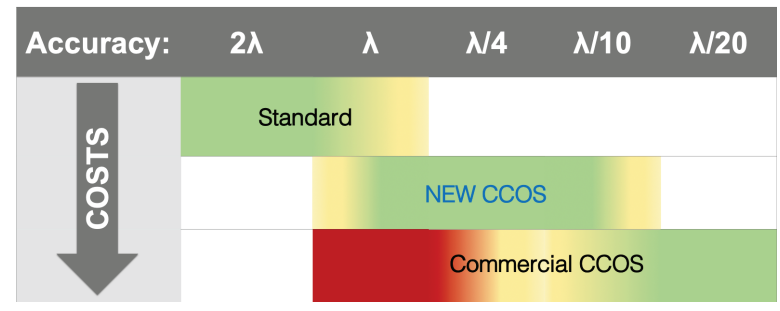

The process is characterised by the "Tool Influence Function", the removal per unit of time as a function of the position within the working area of the process. If the process is linear in time, then the removal at a point on the surface, $R(\vec{x})$, depends on where the process is held on the surface, $\vec{x}_{p}$, how long it is held there, the dwell time $d\left(\vec{x}_{p}\right)$, and the influence function of the process, $f(\vec{u})$,

$$
R(\vec{x})=d\left(\vec{x}_{p}\right) \cdot f\left(\vec{x}-\vec{x}_{p}\right)
$$

If the process is moved over the whole surface of the workpiece along a path $P: \vec{x}_{p}(t)$, which is a smooth curve, then the combined removal becomes

$$
R(\vec{x})=d \otimes f
$$

the convolution of the dwell times with the influence function of the process. The desired removal is equal to the starting surface error $e(\vec{x})$ and a computer algorithm minimizes the predicted residual errors, $r(\vec{x})$,

$$
r=e-d \otimes f
$$

to find the optimal dwell times along the path.

*e-mail: pllow@edmundoptics.com.sg 
The finishing process is using industry standard polishing slurry (e.g. cerium oxide) and polyurethane polishing pads mounted on a spindle. While the pad is moved over the surface, the orientation of the spindle is held normal to the surface being polished, which requires a kinematic system with five degrees of freedom (three for translation and two for orientation).

\section{Results}

A planar work piece of $45 \mathrm{~mm}$ is uniformly polished over a $25 \mathrm{~mm}$ square to evaluate the stability and to estimate the removal rate of the process. Figure 1 shows a 2D slice

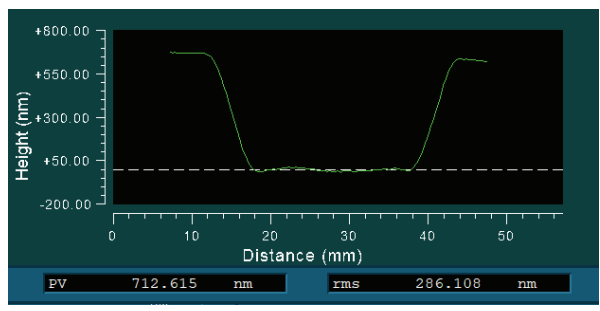

Figure 1. 2D profile through uniform removal square

across the removal area, showing stable removal. The inner area has a uniformity of $10.2 \mathrm{~nm}$ RMS. Based on the volume of removal and total polishing time, an estimated removal rate is established.

After calibrating the removal, corrective polishing was performed on a planar, $50 \mathrm{~mm}$ diameter workpiece with a starting form error of $362 \mathrm{~nm}$ PV and $99 \mathrm{~nm}$ RMS. The
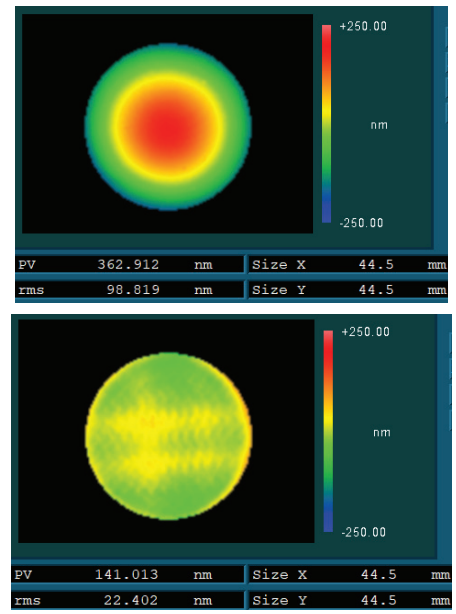

Figure 2. Interferometric measurement of plano before and after correction

resulting form error after corrective polishing is $141 \mathrm{~nm}$ PV and $22 \mathrm{~nm}$ RMS.

The next experiment involves polishing an aspheric lens using a deconvolution algorithm which takes into the account of the TIF of the polishing pad and solves for the optimized dwell time at each polishing point. An $55 \mathrm{~mm}$ diameter aspheric lens is used as the work piece and corrective polishing performance is evaluated within $80 \%$ of the work piece diameter. Starting with an error of $1339 \mathrm{~nm}$ PV and $251 \mathrm{~nm}$ RMS, the corrective polishing is able to bring down the error to $662 \mathrm{~nm}$ PV and $115 \mathrm{~nm}$ RMS. From the metrology data, it is evident that the work
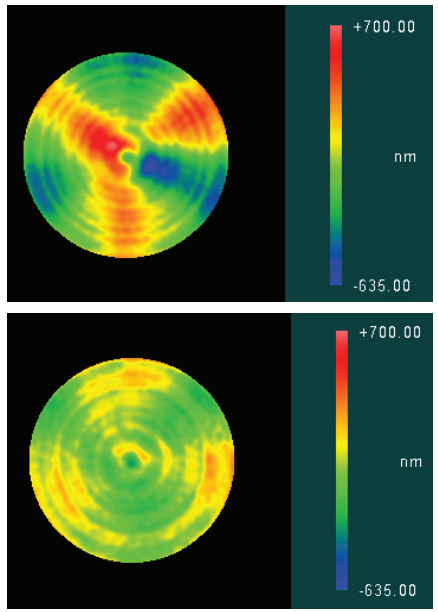

Figure 3. Interferometric measurement of asphere before and after correction

piece has been over-corrected, which results in a slightly inverted error map compared to the input. Hence, this implies that with a better calibrated removal, the correction can be further improved. Moreover, metrology of the aspheric workpiece is performed with a $3 \mathrm{D}$ optical profilometer, with limited accuracy. Further development is currently ongoing using measurement data of a Stitching Interferometer, which has superior accuracy.

\section{Conclusion}

An in-house CCOS system has been designed and built targeting aspheric corrective polishing with an accuracy between $\lambda / 2$ and $\lambda / 10 \mathrm{PV}$. Current results achieved for planar surface is $141 \mathrm{~nm}$ PV and $22 \mathrm{~nm}$ RMS. Initial trials on aspheric surface results in $662 \mathrm{~nm}$ PV and $115 \mathrm{~nm}$ RMS with further development ongoing.

\section{References}

[1] S.D. Jacobs, D. Golini, Y. Hsu, B.E. Puchebner, D. Strafford, I.V. Prokhorov, E.M. Fess, D. Pietrowski, W.I. Kordonski, Magnetorheological finishing: a deterministic process for optics manufacturing, in International Conference on Optical Fabrication and Testing, edited by T. Kasai (SPIE, 1995), Vol. 2576 of Proc. SPIE, pp. 372-382

[2] D. Walker, D. Brooks, A. King, R. Freeman, R. Morton, G. McCavana, S.W. Kim, Opt. Express 11, 958 (2003)

[3] S. Zafran, K.A. Kaufman, M.M. Silver, Optical Engineering 21, 216002 (1982)

[4] R.A. Jones, Appl. Opt. 16, 218 (1977) 